\title{
Cue interaction in human contingency judgment
}

\author{
GRETCHEN B. CHAPMAN and STEVEN J. ROBBINS \\ University of Pennsylvania, Philadelphia, Pennsylvania
}

\begin{abstract}
Most studies of human contingency judgment have been based on the assumption that frequency information about one predictor is assessed in isolation of information about other predictors. Recent evidence, however, suggests that the judged predictive strength of one cue is influenced by the predictive strengths of other copresent cues. Two experiments demonstrate that stimuli with the same outcome contingencies may nonetheless have different predictive strengths as the result of cue interaction. The first experiment, in which a within-subject design was used, provides a demonstration of blocking. A stimulus presented in compound with a strong predictor was rated as less predictive than another stimulus that was presented in compound with a nonpredictive cue. In the second experiment, cue interactions in conditioned inhibition were examined. A stimulus gained negative predictive strength as the result of compound presentations with a positive predictor when the outcome was not presented. This negative predictor was compared with an otherwise analogous stimulus that was not presented in compound with a positive predictor. These results support the use of animal-conditioning models as accounts of human contingency learning.
\end{abstract}

The ability to assess predictive relationships among events in the environment grants humans an obvious benefit. Consequently, the mechanisms underlying such judgments of contingency have become a topic of recent interest (see Alloy \& Tabachnik, 1984, for a review). Most such studies involve giving subjects frequency information about two events. Typically, the information is presented sequentially in a trial-by-trial format. On each trial, either or both of the two events may appear. The subjects are typically asked to rate how predictive the occurrence of one event (the predictor) is with respect to the other event (the outcome). The resulting judgments are then used to infer the underlying structure used by the subjects to store and analyze the frequency information (e.g., Beyth-Marom, 1982; Shaklee \& Tucker, 1980; Ward \& Jenkins, 1965).

One popular idea in this literature has been that subjects store frequency information about pairs of events in a $2 \times 2$ table, where one axis reflects the presence or absence of one event (the predictor) and the second axis reflects the presence or absence of the second event (the outcome) (see Figure 1). By convention, the four cells of such a table are labeled a-d. Cell a stores trials on which both the predictor and outcome occurred, cell $b$ stores trials on which only the predictor occurred, cell $c$ stores trials on which only the outcome occurred, and cell $d$ stores trials on which neither event occurred. Subjects are viewed as performing a mental calculation on

This research was supported by National Science Foundation Grants BNS 83-08176 and BNS 88-03514 to Robert A. Rescorla. The authors thank J. Nachmias of the University of Pennsylvania psychology department for funds for subject payment. Reprint requests should be sent to the first author at the Department of Psychology, University of Pennsylvania, 3815 Walnut Street, Philadelphia, PA 19104. the frequency information stored in this table in order to reach a judgment of the contingency between the two events.

Most researchers interested in contingency judgments (e.g., Beyth-Marom, 1982; Crocker, 1981; Shaklee \& Tucker, 1980; Ward \& Jenkins, 1965) believe that the normative or correct method for forming such a contingency judgment involves comparing the probabilities of the outcome given the presence or absence of the predictor. If the probability of the outcome is greater in the presence of the predictor, then a positive contingency judgment should be made. If the probability of the outcome is lower in the presence of the predictor than in its

\section{$2 \times 2$ TABLE}

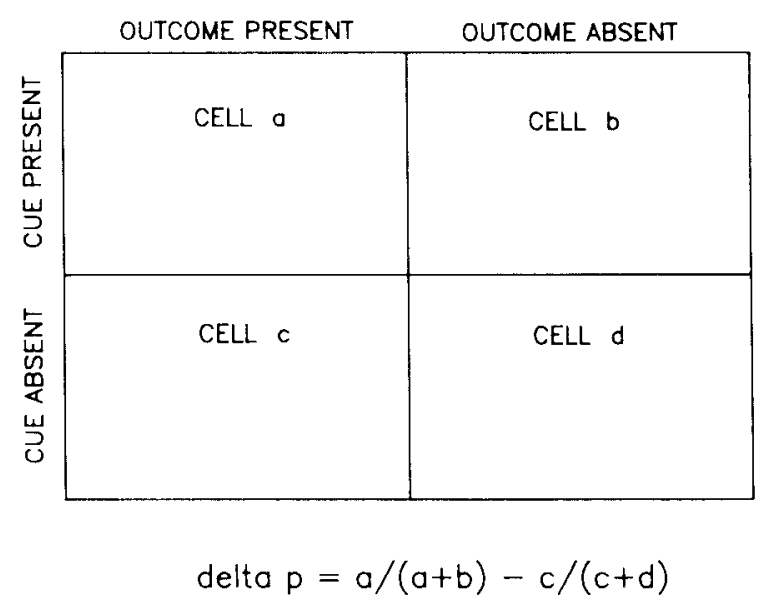

Figure 1. A $2 \times 2$ table and the $\Delta p$ rule of contingency judgment. 
absence, then a negative judgment should result. Put more formally, subjects should calculate the value $a /(a+b)-$ $c /(c+d)$, where $a, b, c$, and $d$ represent the number of instances tabulated in the four cells of the contingency matrix described above. The resulting value is known as $\Delta p$ (delta $p$ ), and hence this strategy is usually called a $\Delta p$ strategy.

A large body of work has accumulated to examine how people solve contingency problems. Although a number of studies have demonstrated contingency judgments that are quite accurate (Wasserman, Chatlosh, \& Neunaber, 1983 ), other studies have concluded that humans use strategies less optimal than the $\Delta p$ rule. Ward and Jenkins (1965), for example, gave subjects information about the frequency of occurrence of two events (cloud seeding and rain) and then asked them to judge the resulting contingency. They concluded from their pattern of results that the subjects inappropriately gave disproportionate weight to information from cell a of the contingency matrix. A number of other investigators have reached a similar conclusion (e.g., Schustack \& Sternberg, 1981; Shaklee, 1983; Shaklee \& Hall, 1983; Shaklee \& Mims, 1982; Shaklee \& Tucker, 1980). Despite these results, the basic assumption that subjects solve such problems by storing the presented information in a $2 \times 2$ frequency table is rarely questioned.

Recently, however, a number of studies have begun to cast doubt on the assumption of information storage in a simple $2 \times 2$ matrix (e.g., Dickinson \& Shanks, 1985; Dickinson, Shanks, \& Evenden, 1984; Gluck \& Bower, 1988; Shanks, 1985b; Shanks \& Dickinson, 1987). These studies involve giving subjects trials on which multiple cues are presented simultaneously. Ratings of the cues in isolation are then collected to see if subjects' evaluations of a signal are affected by the strength of other simultaneously present cues. According to a straightforward contingency matrix theory, judgments for each individual cue should be derived in isolation. Consequently, the predictive value of a cue should not interact with other coincident signals.

The idea behind such studies is derived from research into multiple-stimulus interactions in the animal-learning literature. A number of procedures used in the animallearning laboratory involve simultaneous presentations of multiple stimuli; two such procedures are blocking (e.g., Kamin, 1968, 1969; Rescorla, 1981) and conditioned inhibition (e.g., Pavlov, 1927; Rescorla, 1969; Rescorla \& Holland, 1977). Contingency-judgment research has investigated the blocking procedure (e.g., Shanks, 1985b); however, the conditioned-inhibition procedure has received considerably less attention. Both of these procedures have provided evidence of cue interaction in animal conditioning, and they should therefore prove informative as to the mechanisms underlying human contingency judgment.

Contingency-judgment research on cue interaction has thus far concentrated on the blocking effect first demonstrated by Kamin $(1968,1969)$ in an animal experiment.
In his study, Kamin gave two groups of rats joint presentations of a light and a tone followed immediately by footshock. One of the groups had previously experienced the light signaling shock while the other had not. Despite the identical number of tone-shock pairings in the two groups, Kamin found less conditioned fear to the tone in the group that had undergone prior conditioning. This result seemed to suggest that the pretrained light served to "block" conditioning to the simultaneously presented tone.

Kamin's results suggest that predictive cues are not evaluated in isolation. Instead, the predictive strength of one cue appears to be influenced by the predictive strength of other, copresent cues. Such cue interaction requires a mechanism different from those based on the $2 \times 2$ table.

Another example of cue interaction in animal conditioning comes from studies of conditioned inhibition (e.g., Pavlov, 1927; Rescorla, 1969; Rescorla \& Holland, 1977). A typical inhibition experiment involves teaching the animal that a single predictive stimulus $(P)$ is always followed by the outcome. When $P$ is presented in compound with a second stimulus $(I)$, the outcome is omitted. A variety of assessment procedures can then be used to demonstrate that $I$ has gained negative predictive strength relative to a stimulus presented singly in the absence of the outcome. Thus, $I$ acquires inhibitory strength as a result of its compound presentation with an already established predictor. This paradigm represents another example of two stimuli with identical outcome contingencies that receive different evaluations by the subject.

A number of models have been developed that can account for these basic cue-interaction results. These include associative models (e.g., Mackintosh, 1975; Pearce \& Hall, 1980; Rescorla \& Wagner, 1972), as well as related approaches such as adaptive network models and multiple linear regression models (see Gluck \& Bower, 1988). For the purposes of this paper, the Rescorla-Wagner model will be discussed as a representative of cuecompetition approaches. This model was selected for two reasons. First, it makes easily derived, straightforward predictions about the experiments discussed here. Second, the Rescorla-Wagner model was formulated specifically to address the phenomena of inhibition and blocking. Hence, it provides a more natural and parallel treatment of these two cue-interaction phenomena than do models that grant inhibition less theoretical importance (e.g., Mackintosh, 1975).

According to the Rescorla-Wagner model, simultaneously presented cues compete for predictive power with respect to their outcome. Learning occurs only when there is some discrepancy between the outcome expected on the basis of all currently present signals and the outcome that actually occurs. When the actual outcome is fully expected, no further conditioning results. In the case of Kamin's blocking study, the pretrained light served to fully predict the upcoming shock on the basis of its prior pairings. Consequently, the shock was fully expected following the tone-light compound and little conditioning accrued to the tone. In contrast, the control group had no 
expectation of the shock during its initial tone-light trials and hence showed conditioning to both cues.

The Rescorla-Wagner model also provides a ready explanation of inhibition. On compound trials of a positive predictor $(P)$ and a neutral cue $(I)$, the outcome is expected on the basis of the prior training of $P$. The omission of the outcome thus results in a large negative discrepancy. The predictive strengths of both stimuli are consequently lowered. Because the original strength of $I$ is zero, a decrease causes the predictive strength of $I$ to become negative. Hence, $I$ becomes an inhibitor.

If only one potential predictor occurs before each outcome, then the Rescorla-Wagner model reduces mathematically to the $\Delta p$ formulation (see Appendix). However, the Rescorla-Wagner model, along with other related models, has the additional capability of accounting for competition between multiple cues. The $\Delta p$ model, in contrast, is incapable of predicting such cue competition. Consequently, a clear demonstration of cue competition in human contingency judgments has substantial theoretical importance.

Shanks and his colleagues (Dickinson \& Shanks, 1985; Dickinson et al., 1984; Shanks, 1985b; Shanks \& Dickinson, 1987) have provided some of the best evidence for cue competition in a number of studies of the blocking phenomenon. In these experiments, subjects played a video game requiring them to fire artillery shells at tanks moving through a mine field. The subjects were told to determine the effectiveness of a certain type of shell by firing it at the observed tanks. The tanks could explode when hit by a shell, but they could also explode in the absence of a shell as a result of their hitting a mine.

In the blocking study, some subjects were first given an observation period during which they watched the tanks moving through the mine field. No responses were permitted during this phase, and the subjects observed the tanks exploding with a nonzero probability. Following this phase, the subjects were allowed to fire shells to determine their effectiveness. These subjects assigned a lower rating regarding the effectiveness of the shells than did the subjects who did not experience the observation period. These results appear consistent with cuecompetition models. For the first group, the mine field gained predictive strength during the observation period. During the subsequent phase, the predictive value of the mine field was sufficient to block learning about the shells, causing them to receive a low rating. In contrast, the second group did not have an observation period during which the mine field could gain predictive strength. Thus, the mine field was not able to block learning about the artillery shells.

In several studies, Shanks and his colleagues (Dickinson et al., 1984, Experiment 2; Shanks, 1985b, Experiment 1) have been careful to equate the normative $\Delta p$ for both the blocking and control conditions. However, these designs required that the frequencies stored in each cell of the $2 \times 2$ table were not identical for the two conditions. Because the blocking condition involved added ex- perience with the outcome in the absence of the predictor, the blocking $2 \times 2$ table contained a higher proportion of observations in the $c$ and $d$ cells. Although the different ratings of the predictor in the blocking and control condition cannot be explained by the use of the normative $\Delta p$ strategy, they could be explained by the use of a nonnormative $2 \times 2$ table strategy, such as those described by Shaklee and Hall (1983). Thus, cuecompetition models need not be invoked to explain these results. What is clearly required, therefore, is a design in which the blocked and control stimuli have identical $2 \times 2$ tables associated with them.

Furthermore, although reliable and replicable, the blocking result in many of these studies is small. The difference between the blocking group and the control group averages about 6 points on a $0-100$ rating scale. The modest magnitude of the effect suggests that the result may not be replicated in other procedures. Moreover, the procedure used by Shanks and his colleagues (Dickinson et al., 1984; Shanks, 1985b) involved a relationship between a subject's response and the outcome. Other contingency-judgment studies (e.g., Beyth-Marom, 1982; Schustack \& Sternberg, 1981; Shaklee \& Hall, 1983; Shaklee \& Mims, 1982; Shaklee \& Tucker, 1980; Ward and Jenkins, 1965) have typically examined relationships between a stimulus and the outcome. Shanks's subjects chose when to fire a shell and when to withhold a shell; thus they were able to make explicit tests of the shell's effectiveness. It may be that such a task is especially suited to the demonstration of blocking. Observation of blocking in a stimulus-outcome design would broaden the implications of that result.

Gluck and Bower (1988) collected data in a study examining stimulus-outcome relationships which also suggest the presence of blocking in human contingency judgments. In their study, subjects were given information about a number of symptoms that predicted the presence of one of two mutually exclusive diseases. The subjects were then asked to rate the probability of either disease given the presence of a given symptom. Ratings assigned to the symptoms in one experiment were compared with ratings assigned to those same symptoms in a second experiment which also included other highly predictive cues. Symptom ratings were reduced as a result of the simultaneous presence of these highly predictive cues. This result could be interpreted as blocking. A plausible alternative explanation, however, is that the presence of the highly predictive cues prompted a change in the use of the rating scale in the second experiment. The subjects may have felt it was necessary to lower the ratings for the putatively blocked symptoms in order to allow the rating scale to accommodate the high ratings warranted by the predictive cues.

Therefore, while there is growing evidence to suggest that blocking occurs in human contingency judgments, no single study has yet demonstrated the phenomenon in a wholly unconfounded manner. Furthermore, there is little evidence as to whether other examples of cue interac- 
tion from the animal-conditioning literature can similarly be demonstrated in human contingency learning. For example, only limited work has been done to demonstrate conditioned inhibition in contingency-judgment tasks (e.g., Shanks, 1987).

Consequently, the present studies were designed to accomplish two goals. First, Experiment 1 was intended to demonstrate blocking in a contingency-judgment design free of many of the interpretative difficulties discussed earlier. To this end, a within-subject design recommended by Rescorla (1981) as providing the most unconfounded demonstration of blocking was employed. Second, Experiment 2 was intended to demonstrate the phenomenon of conditioned inhibition in a contingency-judgment task employing a similar within-subject design. Sixteen subjects participated in both of these experiments.

\section{EXPERIMENT 1}

The task used in this study involved asking subjects to use changes in the price of individual fictional stocks to predict the behavior of a fictional stock market. An increase in the price of a stock corresponded to the presence of a predictor, whereas no increase in the price of a stock corresponded to the absence of a predictor (prices never showed decreases). Similarly, an increase in the value of the entire market corresponded to the presence of the outcome, whereas no increase in the value of the entire market corresponded to the absence of the outcome.

Information about the stock prices and value of the market were presented in a series of trials. These trials were divided into two phases. In the first phase, whenever one stock $(\mathrm{P})$ rose in price, the market rose in value as well. Whenever a second stock $(\mathrm{N})$ rose in price, the market failed to increase in value. Thus, stock $P$ was a positive predictor, whereas stock $\mathrm{N}$ was nonpredictive. In the second phase, pairs of stocks showed price increases. On some trials, stocks $\mathrm{P}$ and $\mathrm{B}$ rose together and consistently predicted a market increase. On other trials, stocks $\mathrm{N}$ and $\mathrm{C}$ rose together and consistently predicted a rise in the value of the market. Periodically, the subjects were asked to assess the predictive value of each stock.

If copresent cues compete with each other for predictive power, then stock B (the blocked stimulus) should receive a lower rating than stock $C$ (the control stimulus). One advantage of this within-subject design is the identical objective contingencies for stocks $B$ and $C$. When calculating a contingency matrix for stock $\mathrm{B}$, for example, the number of outcomes in the absence of stock B would include those outcomes following $\mathrm{P}$ alone and those following the NC compound. This number is identical to the number of outcomes in the absence of stock C. Similarly, each of the other three cells of the contingency matrix is identical for stocks B and C. Thus, if subjects rate individual cues in isolation, stocks $\mathrm{B}$ and $\mathrm{C}$ should receive identical ratings.

\section{Method}

Subjects and Apparatus. The subjects were 16 male and female undergraduate students. They were run in individual sessions using IBM PC computers and were paid $\$ 5$ for their participation in this experiment and in Experiment 2.

Procedure. When the subjects first sat down at the computer, instructions were present on the screen explaining that they would be shown information on fictional stock prices over several months while being asked to make predictions about the fictional market as a whole. They were also instructed that they would periodically be asked to rate the predictive value of each stock, and that they should base their judgments on all the information they had received up to that point. They were further told that stocks could have three kinds of predictive value. Positive predictors were defined as stocks whose rise made the market as a whole more likely than usual to rise. Negative predictors were defined as stocks whose rise signaled that the market was less likely than usual to rise. Finally, stocks whose rise provided no novel information about the market were defined as nonpredictors.

After the instructions, a display of four stocks appeared on the screen. A date that progressed with each trial was displayed at the top of the screen. The names of four fictitious corporations were listed. These names were either Alastair Corp., Beatrice Corp., Constance Corp., and Duncan Corp. (set 1) or Roland Corp., Samantha Corp., Theodore Corp., and Ursula Corp. (set 2). Beside each name was the change in price for that corporation: either the word $u p$ in uppercase flashing green letters or the word same in lowercase nonflashing red letters.

Below the stock listing was printed the question, "Will the market go up in value tomorrow or not? Press $Y$ if the market will go up tomorrow, $\mathrm{N}$ if it will not." After responding, each subject was asked to indicate how confident she or he was about the prediction by giving a number from 1 to 5 , where 1 meant not confident and 5 meant very confident. The word "correct" or "incorrect" was then printed on the screen to indicate the accuracy of the subject's yes or no response. In addition, the cumulative percent correct was printed on the screen. This display remained on the screen for $2 \mathrm{sec}$ before all but the stock listings were erased; the stock listing was displayed with the correct answer ("The market goes up" or "The market remains the same"). After the subject pressed a key to continue, the next trial was displayed.

Periodically during the session, the subject was asked to rank and rate the four stocks according to their predictive values. At such a point, the subject received instructions to rank the stocks from 1 to 4 , where 1 indicated the most positive predictor and 4 indicated the most negative predictor. Then the subject was asked to rate the predictive value of the stocks by assigning each one a number from +100 to -100 , where +100 indicated a perfect positive predictor, -100 indicated a perfect negative predictor, and 0 indicated a random nonpredictor. After the subject filled in the ratings for the four stocks, the program continued with the next trial.

Each subject was run through two different sessions, one for Experiment 1 and another for Experiment 2. After the first session, the subject waited while the experimenter set the program for the second session. After completion of the second session, each subject was paid. The order of the sessions in Experiments 1 and 2 was randomized across subjects. In addition, use of the corporation names in sets 1 and 2 was counterbalanced across the two sessions. Which corporation name corresponded to which stimulus role was also counterbalanced across subjects.

The different roles that the stocks played are indicated by the letters P, N, B, and C. This experiment contained three phases: element training, compound training, and test trials.

The element-training phase consisted of 36 trials. On 12 of these trials, only stock $P$ went up and the market also went up (abbreviated as $\mathrm{P}+$ trials). There were also 12 trials in which only stock $\mathrm{N}$ went 
up and the market never went up ( $N-$ ). On the other 12 trials, no stocks went up and the market did not go up. These three trial types were intermixed in this first phase; following the 36 th trial, subjects were asked to rank and rate the four stocks.

The second phase consisted of 72 trials divided into two 36-trial blocks. In each block, the subjects received 12 of each of three trial types. On some trials, both stock $P$ and stock $B$ went up and the market always went up $(\mathrm{PB}+)$. On other trials, both stock $\mathrm{N}$ and stock $\mathrm{C}$ went up and the market always went up $(\mathrm{NC}+)$. On the remaining trials, no stocks went up and the market never went up. The three trial types were intermixed within each block; after each block, the subjects were asked to rank and rate the four stocks.

In the final phase, the subjects were given 12 trials in which only one stock went up per trial and the market never rose. Each of the four stocks was presented three times in this phase. Estimates given on these trials were used as an additional measure of the predictive strength of each stock.

\section{Results and Discussion}

Three measures were used to compare the judged predictive value of the stocks. First, the subjects ranked the stocks from 1 to 4 . Second, the subjects rated the stocks by assigning each one a number from -100 to +100 . Third, the subjects made predictions about the market as a whole and gave confidence ratings on the individual test trials.

Figure 2 presents the data from the subjects' ratings of the stocks. The left half of the figure shows the mean rating for each stock given after the first phase of the experiment. Planned contrasts indicated that $P$ was rated higher than $\mathrm{N}$ [Wilcoxon $T(14)=0, p<.01$ ], whereas stocks $\mathrm{B}$ and $\mathrm{C}$ did not differ from one another $[T(3)=$ $0, p>.05]$. This pattern was expected because $\mathrm{P}$ was presented as a positive predictor whereas $\mathrm{N}$ was presented as a nonpredictor. This difference in predictive strengths is important, because it enables $P$ and $N$ to compete differentially with $\mathrm{B}$ and $\mathrm{C}$ in the second phase. Neither stock $B$ nor stock $C$ had thus far shown increases in price; they were therefore expected to be rated similarly.

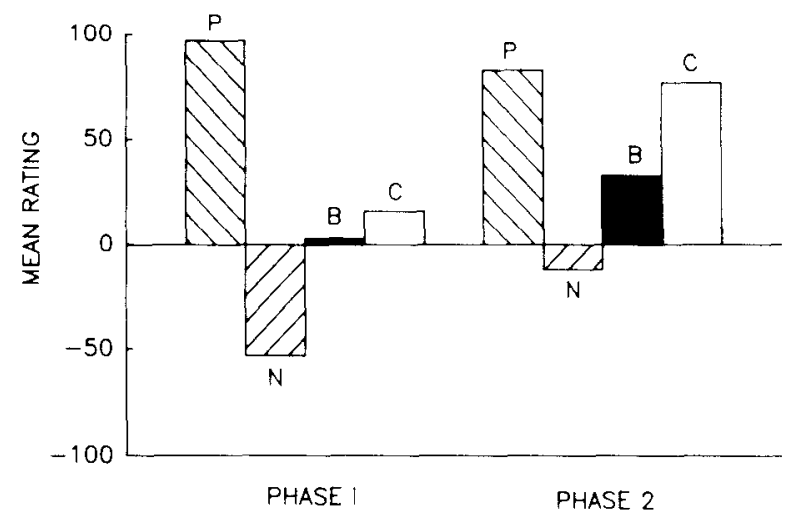

Figure 2. Subjects' ratings given in Experiment 1 for the four stocks. In Phase 1, rises in the price of stock $P$ were consistently followed by rises in the price of the market, whereas rises in the price of stock $\mathbf{N}$ were consistently followed by no changes in the market. In Phase 2, stocks $P$ and $B$ rose in price together and were followed by increases in market value; stocks $\mathrm{N}$ and $\mathrm{C}$ similarly rose together and were followed by a rise in price of the market.
Additional post hoc comparisons demonstrated that $\mathrm{P}$ was rated higher than $\mathrm{B}[T(16)=0, p<.01]$ and $\mathrm{C}$ $[T(14)=0, p<.01]$. Stoxk $N$ was rated lower than stock $\mathrm{B}|T(12)=12, p<.05|$ and stock $\mathrm{C} \mid T(12)=0$. $p<.011$. It is interesting that stock $\mathrm{N}$ was actually rated lower than stocks $B$ and $C$. Such a result appears inconsistent with the Rescorla-Wagner model, which predicts that all three stimuli should remain neutral. However, the Rescorla-Wagner model can accommodate these results if one makes the added assumption that $B$ and $C$ gained some generalized positive strength from $P$. Stimulus $N$ may have been protected from this generalization by the trials on which its rise predicted no rise in the market. Therefore, the subjects may have viewed $N$ as having no predictive strength and $B$ and $C$ as having a modest positive level. Note that this argument implies that subjects utilized their own arbitrary zero point, since $N$ received a negative rating

The right half of Figure 2 shows the mean rating for each stock during the compound-training phase. The two sets of ratings collected during this phase were combined for the purposes of analysis. Planned contrasts indicated that stock $\mathrm{P}$ continued to be rated higher than stock $\mathrm{N}$ $[T(13)=0, p<.01]$. Of primary interest, stock B was rated lower than stock $\mathrm{C}[T(9)=0, p<.01]$. Ranking data pooled over the compound-training phase confirmed the basic blocking result. Eleven of 16 subjects ranked $\mathrm{C}$ as more positively predictive than $\mathrm{B}$ during this phase (there were two ties due to pooling over blocks). A sign test $(p<.05)$ showed this difference between $C$ and B was reliable.

The test-trial data yielded a similar pattern of results. For the first test trial of each stock, each subject's prediction of whether or not the market would go up was converted to $a+1$ if the response was yes or $a-1$ if the response was no. This value was multiplied by the confidence rating for that trial, a number from 1 to 5 . By using this measure, stock $B$, with a value of 0.1 , was assessed as reliably less predictive than stock $C$, with a value of $3.4[T(13)=13.5, p<.05]$.

This consistent pattern of lower ratings for stock $B$ than for stock $C$ constitutes the blocking effect. Since stocks $B$ and $C$ received identical outcomes during all phases of the study, their differential ratings can only be attributed to the differential predictive strength carried by their $P$ and $\mathrm{N}$ partners. It is important to notice that no $2 \times 2$ matrix strategy can account for such a result. To apply this storage format, a separate table would have to be constructed for each stock. A given table would store the presence or absence of the outcome and one of the predictors, ignoring the presence of other cues. In this framework, any measure of the contingency between stock $B$ and the outcome would be the same as that between stock $C$ and the outcome, since the frequency scores in the $2 \times 2$ table are identical for the two predictors.

By contrast, the results of Experiment 1 are quite consistent with cue-competition models such as the RescorlaWagner model. As predicted by such models, copresent 
cues are not evaluated in isolation but rather compete for predictive strength. Therefore, contingency judgments reflect more than the statistical relatedness between signal and outcome. As do animals in conditioning studies, humans appear to judge the degree of novel information carried by a cue when assessing its predictive properties.

\section{EXPERIMENT 2}

Although human contingency-judgment studies have focused almost exclusively on the blocking result, a number of other examples of cue interaction have been well established in the animal laboratory. As described in the introduction, one such example is the conditioned inhibition procedure first studied by Pavlov (1927). Experiment 2 was intended to demonstrate this phenomenon in a human contingency-judgment task.

As in Experiment 1, the design had subjects compare two stimuli with identical outcome contingencies. In this case, neither cue was ever paired with the outcome. One stimulus always appeared in compound with a previously positive predictor, whereas the other stimulus always appeared on its own. The Rescorla-Wagner model anticipates that the stimulus presented in compound will accrue negative predictive strength compared with the cue presented alone. A $2 \times 2$ matrix model would predict equal ratings for the two stimuli.

This study also served to test a second prediction of the Rescorla-Wagner model. To explain contingency sensitivity in animals, the Rescorla-Wagner model postulates the presence of a context stimulus. The context stimulus comprises the constant features of the environment and is assumed to be present on every trial. The presence of such a context stimulus is quite useful in explaining contingency results such as those reported by Shanks, among others (Baker, Berbrier, \& Vallee-Tourangeau, 1989; Dickinson et al., 1984; Shanks, 1985a). Shanks found that the perceived predictive strength of a cue was reduced by the presence of unsignaled outcomes. According to the Rescorla-Wagner model, these unsignaled outcomes were actually associated with the context. Therefore, the context acquired predictive strength and successfully blocked learning about the discrete cue occurring in its presence.

If all of the outcomes in a given session are signaled by explicit cues, however, then the context should attain a neutral strength due to its failure to provide novel information. To test this prediction, the current study employed one stock $(\mathrm{X})$ which played the role of context; its price rose on every trial. The Rescorla-Wagner model predicts that this context cue will have no predictive strength. Thus, the context stimulus should not differ from the control stimulus presented repeatedly in the absence of an outcome. As discussed above, both stimuli should receive higher ratings than should the cue given conditioned-inhibition training.

Experiment 2 contained two phases. In the first phase, stock $P$ was established as a positive predictor of an increase in the market's value. The context stock (X) rose in price on trials in which $\mathrm{P}$ also rose $(\mathrm{XP}+)$. However, $X$ also rose in price on other trials in which the market did not change in value $(\mathrm{X}-)$. In the second phase, the two trial types from Phase 1 were continued. In addition, trials in which $P, X$, and a third stock (I) rose in price resulted in no change in the market's value (XPI-). As a result of this training, stock I was expected to gain negative predictive (inhibitory) strength. On still other trials, stock $\mathrm{X}$ and another stock $(\mathrm{N})$ rose in price and the market did not change in value ( $\mathrm{XN}-$ ). Stock $\mathrm{N}$ was anticipated to remain a nonpredictor of the outcome since it did not co-occur with a highly predictive cue. If stock I proved to be a conditioned inhibitor, it would be rated lower than both $\mathrm{N}$ and $\mathrm{X}$.

This design directly compares predictions from the Rescorla-Wagner model with those of contingency-matrix models. The Rescorla-Wagner model predicts here that two stimuli with identical outcome contingencies (I and $\mathrm{N})$ should nevertheless receive different ratings. By contrast, two stimuli with different outcome contingencies ( $\mathrm{X}$ and $\mathrm{N}$ ) are expected to receive similar evaluations. Contingency-matrix models make the opposite prediction.

\section{Method}

Subjects and Apparatus. The subjects were the same 16 subjects used in Experiment 1. In addition, the same computer program was used, with the exception of the particular trial types administered.

Procedure. The instructions given were the same as those in Experiment 1 . The details of trial presentation and elicitation of ratings and rankings were also the same.

Experiment 2 was divided into two phases. The first phase consisted of a total of 20 trials. On 10 of those trials, only stock $X$ went up and the market did not go up $(\mathrm{X}-)$. On the other 10 trials, stocks $\mathrm{X}$ and $\mathrm{P}$ both went up and the market rose as well $(\mathrm{XP}+)$. These two trial types were intermixed in the first phase; the subjects were asked to rank and rate the four stocks after the last trial.

The second phase consisted of 120 trials and was divided into three blocks of 40 trials each. Each block consisted of four trial types occurring 10 times each. On some trials, only stock X rose, and the market stayed the same $(X-)$. On other trials, stocks $X$ and $\mathrm{P}$ both rose, and the market went up as well $(\mathrm{XP}+)$. The third trial type involved a rise in stocks $\mathrm{X}, \mathrm{P}$, and I followed by no change in the market (XPI-). Finally, stocks $X$ and $N$ rose together on some trials and were followed by no change in the market $(\mathrm{XN}-$ ). The four trial types were intermixed within each block. After each of the three 40-trial blocks in this phase, the subjects were asked to rank and rate the 4 stocks using the procedure described in Experiment 1 .

\section{Results and Discussion}

Two measures were used in this experiment to compare the judged predictive value of the stocks: relative rankings on the 1 to 4 scale and absolute ratings on the +100 to -100 scale. Figure 3 depicts the rating results. The left half of the figure shows the mean rating for each stock after the first phase of the experiment. Stock P was rated higher than stock $\mathrm{X}[T(15)=0, p<.01]$. Stocks $\mathrm{I}$ and $\mathrm{N}$ did not differ from one another $[T(2)=0$, $p>.05]$. Thus it appears that $\mathrm{P}$ was successfully established as highly predictive of the market's increase. Since 


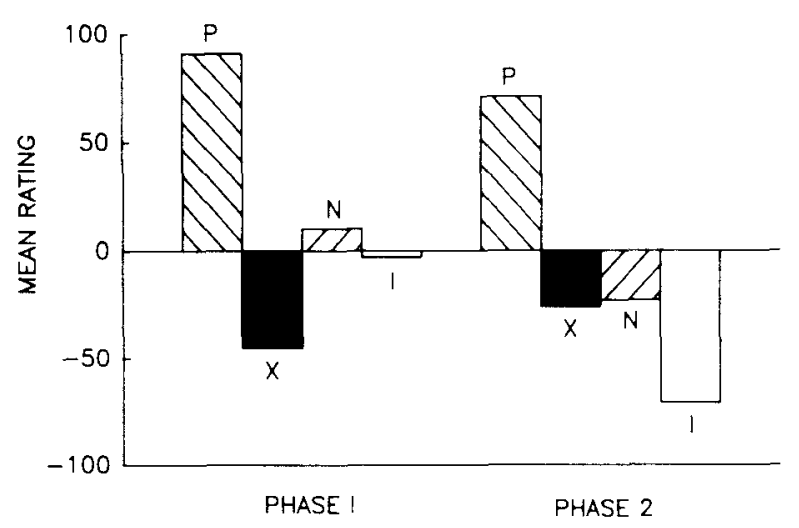

Figure 3. Subjects' ratings given in Experiment 2 for the four stocks. In Phase 1 , rises in the price of stock $X$ alone were followed by no change in the market, whereas increases in stock $X$ together with increases in stock $P$ were always followed by a rise in price of the market. In Phase 2, the same two trial types were presented in addition to trials in which stocks $X, P$, and I rose together, followed by no change in the market and trials in which stocks $X$ and $\mathrm{N}$ rose together, followed by no change in the market.

neither I nor $\mathrm{N}$ had yet risen in price, it was expected that their ratings should not differ from one another.

Post hoc comparisons indicated that stock $P$ was rated higher than both $\mathrm{N}[T(14)=0, p<.01]$ and $\mathrm{I}[T(15)$ $=0, p<.01]$. Stock X was rated lower than I $[T(13)$ $=15.5, p<.05]$ and lower than $\mathrm{N}[T(12)=3$, $p<.01]$. This result is similar to that seen with stimulus $\mathrm{N}$ in the first phase of Experiment 1 .

The right half of the figure shows mean ratings for the second phase of the experiment. The ratings given after each of the three blocks in this phase were combined for the purposes of analysis. Stock I was rated lower than both stock $\mathrm{N}[T(11)=0, p<.01]$ and stock $\mathrm{X}[T(15)=15$, $p<.01]$. The ratings of stocks $\mathrm{N}$ and $\mathrm{X}$ did not differ $[T(13)=44, p>.05]$.

The ranking data revealed a similar pattern of results. The rankings given after each of the three blocks of the second phase were pooled. By using this combined measure, 10 of 16 subjects ranked I below both $\mathrm{N}$ and $\mathrm{X}$ (there were two ties) (binomial test, $p<.01$ ). Stocks $\mathrm{N}$ and $\mathrm{X}$ were not rated differentially, as only 9 of 14 subjects rated $\mathrm{X}$ higher than $\mathrm{N}$ (sign test, $p>.05$ ).

These results are fully consistent with predictions made by the Rescorla-Wagner model. As a result of failing to receive the outcome when they otherwise expected it on XPI - trials, the subjects endowed stock I with inhibitory strength. The presence of such negative value can be inferred from the lower rating given stock I than stock $N$. Since a rise in $\mathrm{N}$ never predicted a rise in the market, $\mathrm{N}$ should have received no positive strength. The reliably lower rating of I indicated that it must have acquired negative value.

Other studies (Baker, Berbrier, \& Vallee-Tourangeau, 1989; Dickinson \& Shanks, 1985; Dickinson et al., 1984; Shanks, 1985a; Shanks, 1987; Shanks \& Dickinson, 1987) have used designs that could result in the accrual of nega- tive predictive strength to cues. For example, Shanks (1987) presented subjects with a procedure in which the outcome was more likely in the absence of the predictor than in the presence of the predictor. Ratings of this negative predictor dropped progressively across trials and achieved a negative value below that achieved with a zero contingency. However, this negative predictor was not compared with a nonpredictive stimulus with the same objective $\Delta p$. Therefore, although Shanks's study demonstrates the acquisition of negative predictive strength on the basis of a negative $\Delta p$, it fails to demonstrate inhibition generated by cue interaction. The present study provides the first such demonstration by comparing a negative predictive cue with a nonpredictive cue with the same $\Delta p$.

In addition to establishing the basic inhibition phenomenon, these results confirm a further prediction of the Rescorla-Wagner model. Despite their very different training histories, stocks $X$ and $N$ received nondifferential ratings that were intermediate to those given to stocks $P$ and I. According to the Rescorla-Wagner model, the treatment of stock $X$ should have left it with zero strength. On XP compound trials, learning about $X$ would be blocked by the presence of the predictive stock P. If $X$ did acquire any strength, that strength would be lost on the $\mathrm{X}$ - trials. Stock N should have remained neutral as well. Since its price always rose in conjunction with neutral stock $X$ and predicted no change in the market, no discrepancy between expectation and outcome should have occurred on such trials.

It is important to notice that stocks I and $\mathrm{N}$ should have been identically assessed if evaluations of different cues occur in isolation. As in Experiment 1, the contingencies between each of the primary comparison stimuli and the outcome were the same. Therefore, the reliably different evaluation of these two cues could only result from the influence of the other cues presented on the compound trials. Furthermore, stocks $X$ and $N$ received identical ratings despite their different individual contingencies with the outcome. Hence, two stimuli with identical outcome contingencies (I and $\mathrm{N}$ ) received differential ratings, whereas two stimuli with different outcome contingencies ( $\mathrm{X}$ and $\mathrm{N}$ ) were given similar evaluations. These results provide further evidence against the idea that contingency judgments result from the storage and evaluation of information in individual $2 \times 2$ matrices. Instead, the judgments recorded here appear consistent with a cuecompetition model such as that proposed by Rescorla and Wagner.

\section{GENERAL DISCUSSION}

The two studies reported here provide clear evidence of interactions between simultaneously presented cues in a contingency-judgment task. The results demonstrate that the evaluation of a signal is not determined solely by its contingency with the outcome, but rather by its ability to provide novel or surprising information about that out- 
come. In this respect, the rules governing contingency judgments in humans and classical conditioning in animals seem strikingly similar.

Experiment 1 demonstrated the presence of competition for predictive strength between two simultaneously presented stimuli. In that study, a novel cue signaling an outcome in the presence of a positive predictor gained less predictive power than a novel cue placed in compound with a poor predictor. In other words, subjects receiving two stimuli with identical outcome contingencies nevertheless evaluated those cues differently. These results are inconsistent with the idea that subjects form contingency judgments by storing and analyzing $2 \times 2$ tables of frequency information for each signal in isolation.

Similar results were obtained from Experiment 2. Once again, two stimuli with identical outcome contingencies were given differential ratings by the subjects. In this case, one stimulus acquired negative strength as a result of its signaling the absence of an outcome when presented simultaneously with a positive predictor. A comparison stimulus which singly predicted the absence of an outcome failed to acquire such inhibitory properties. Furthermore, this study demonstrated that two stimuli with different outcome contingencies received similar ratings. Both the neutral comparison cue and the context cue were rated identically, as was predicted by the Rescorla-Wagner model.

Some recent results reported by Shanks $(1986,1989)$ are consistent with these findings as well. In one study using the tank video game described in the introduction, Shanks gave two groups of subjects experience with a random contingency between a predictor and the outcome. For one group, however, the outcomes occurring in the absence of the predictor were signaled by a second cue. The subjects in this signaled group rated the target predictor higher than did the subjects in the unsignaled group. As in the present studies, two stimuli with identical outcome contingencies were rated differentially. The Rescorla-Wagner model quite easily accounts for Shanks's results, since signaling the extra outcomes should have blocked the ability of the context to become a good predictor. This weaker context was then less capable of interfering with learning about the target predictor.

These results indicate that any successful theory of human contingency judgment must incorporate cue interaction. The Rescorla-Wagner model is one theory capable of explaining these results; however, other models are also able to account for phenomena such as blocking and conditioned inhibition. Gluck and Bower (1988) have pointed out that simple adaptive network models, as well as multiple linear regression models, are formally similar to the Rescorla-Wagner model and are thus capable of predicting the current results. In addition, other models of animal learning (e.g., Mackintosh, 1972; Pearce \& Hall, 1980), although quite different in mechanism from the RescorlaWagner model, also predict cue interactions.

A number of recent reviews have suggested that principles derived from human-contingency-judgment studies might serve to alter our frameworks for understanding animal conditioning (Alloy \& Tabachnik, 1984; Holyoak, Koh, \& Nisbett, 1989). The results of the present studies seem to suggest a different approach, however. There are well-specified conditioning models that focus on the role of cue interactions in associative learning (e.g., Mackintosh, 1975; Pearce \& Hall, 1980; Rescorla \& Wagner, 1972). Increasing evidence for the importance of cue interactions in human contingency judgments suggests that such conditioning models warrant further attention.

\section{REFERENCES}

Alloy, L. B., \& TABACHNik, N. (1984). Assessment of covariation by humans and animals: The joint influence of prior expectation and current situational information. Psychological Review, 91, 112-149.

Baker, A. G., Berbrier, M. W., \& Vallee-Tourangeau, F. (1989). Judgments of a $2 \times 2$ contingency table: Sequential processing and the learning curve. Quarterly Journal of Experimental Psychology, 41B, 65-97.

Beyth-Marom, R. (1982). Perception of correlation reexamined. Memory \& Cognition, 10, 511-519.

Crocker, J. (1981). Judgment of covariation by social perceivers. $P$ sychological Bulletin, 90, 272-292.

Dickinson, A., \& Shanks, D. (1985). Animal conditioning and human causality judgment. In L. G. Nilsson \& T. Archer (Eds.), Perspectives on learning and memory (pp. 167-191). Hillsdale, NJ: Erlbaum.

Dickinson, A., Shanks, D., \& Evenden, J. (1984). Judgment of actoutcome contingency: The role of selective attribution. Quarterly Journal of Experimental Psychology, 36A, 29-50.

GLUCK, M. A., \& Bower, G. H. (1988). From conditioning to category learning: An adaptive network model. Journal of Experimental Psychology: General, 117, 227-247.

Holyoak, K. J., KOH, K., \& Nisbett, R. E. (1989). A theory of conditioning: Inductive learning within rule-based default hierarchies. Psychological Review, 96, 315-340.

KAMIN, L. J. (1968). 'Attention-like' processes in classical conditioning. In M. R. Jones (Ed.), Miami symposium on the production of behavior: Aversive stimulation (pp. 9-33). Coral Gables, FL: University of Miami Press.

Kamin, L. J. (1969). Predictability, surprise, attention, and conditioning. In B. Campbell \& R. Church (Eds.), Punishment and aversive behavior (pp. 279-296). New York: Appleton.

MACKINTOSH, N. J. (1975). A theory of attention: Variations in the associability of stimuli with reinforcement. Psychological Review, 82 , 276-298

Pavlov, I. P. (1927). Conditioned reflexes. Oxford: Oxford University Press.

Pearce, J. M., \& Hall, G. (1980). A model for Pavlovian learning: Variations in the effectiveness of conditioned but not of unconditioned stimuli. Psychological Review, 87, 532-552.

Rescorla, R. A. (1969). Pavlovian conditioned inhibition. Psychological Bulletin, 72, 77-94

RESCORLA, R. A. (1981). Within-signal learning in autoshaping. Animal Learning \& Behavior, 9, 245-252.

Rescorla, R. A., \& Holland, P. C. (1977). Associations in Pavlovian conditioned inhibition. Leaming \& Motivation, 8, 429-447.

Rescorla, R. A., \& Wagner, A. R. (1972). A theory of Pavlovian conditioning: Variations in the effectiveness of reinforcement and nonreinforcement. In A. H. Black \& W. F. Prokasy (Eds.), Classical conditioning II: Current theory and research (pp. 64-99). New York: Appleton-Century Crofts.

Schustack, M. W., \& Sternberg, R. J. (1981). Evaluation of evidence in causal inference. Joumal of Experimental Psychology: General, 110, 101-120.

ShakleE, H. (1983). Human covariation judgment: Accuracy and strategy. Learning \& Motivation, 14, 433-448.

ShakleE, H., \& Hall, L. (1983). Methods of assessment strategies 
for judging covariation between events. Journal of Educational $P$ sychology, 75, 583-594.

Shaklee, H., \& Mims, M. (1982). Sources of error in judging event covariations: Effects of memory demands. Journal of Experimental Psychology: Learning, Memory, \& Cognition, 8, 208-224

ShaKLEE, H., \& TUCKER, D. (1980). A rule analysis of judgments of covariation between events. Memory \& Cognition, 8, 459-467.

Shanks, D. (1985a). Continuous monitoring of human contingency judgment across trials. Memory \& Cognition, 13, 158-167.

SHANKS, D. (1985b). Forward and backward blocking in human contingency judgment. Quarterly Journal of Experimental Psychology, 37B, $1-21$

Shanks, D. (1986). Selective attribution and the judgment of causality. Learning \& Motivation, 17, 311-334.

ShaNKS, D. (1987). Acquisition functions in contingency judgment. Learning \& Motivation, 18, 147-166.

Shanks, D. (1989). Selectional processes in causality judgments. Memory \& Cognition, 17, 27-34.

Shanks, D. , \& Dickinson, A. (1987). Associative accounts of causality judgment. In $\mathrm{H}$. Bower (Ed.), The psychology of learning and motivation: Advances in research and theory, 21 (pp. 229-261). New York: Academic Press.

WARD, W. C., \& Jenkins, H. M. (1965). The display of information and the judgment of contingency. Canadian Journal of Psychology, 19, 231-241

Wasserman, E. A., Chatlosh, D. L., \& Neunaber, D. J. (1983). Perception of causal relations in humans: Factors affecting judgment of response-outcome contingencies under free-operant procedures. Learning \& Motivation, 14, 406-432.

\section{APPENDIX}

When only one predictor and one outcome are involved, the Rescorla-Wagner model reduces to the $\Delta p$ rule. This proof is derived from two important assumptions of the Rescorla-Wagner model. First, in addition to the explicit cue considered, a context cue is present on every trial. Second, contingency learning will continue until the discrepancy between the actual and expected outcomes is zero.

The learning rule for the Rescorla-Wagner model is:

$$
\Delta V_{i}=\alpha B\left(\lambda_{j}-\Sigma V_{k}\right) .
$$

$V_{i}$ is the predictive strength of cue $i . \Delta V_{i}$ is the change in predictive strength for cue $i$ as a result of the current trial. $\alpha B$ is a rate parameter that depends on the salience of cue $i$ and outcome $j . \lambda_{j}$ is the actual outcome of the trial. If the outcome is present, this value is 1 ; if the outcome is absent, this value is $0 . \Sigma V_{k}$ is the expected outcome or the sum of the predictive strengths of all cues present on that trial.

In a contingency-judgment experiment involving one cue, there are four types of trials corresponding to the four cells of the $2 \times 2$ table. If $X$ represents the context and A represents the cue, then these trial types are $X A+, X A-, X+$, and $X-$, which correspond to cells $a, b, c$, and $d$, respectively. On each trial, one equation is constructed for each of the cues (including the context) present on that trial. Listed below are the equations associated with each of the trial types.

$X A+$ trials:

$$
\begin{aligned}
& \Delta V_{X}=\alpha B\left[1-\left(V_{X}+V_{A}\right)\right] \\
& \Delta V_{A}=\alpha B\left[1-\left(V_{X}+V_{A}\right)\right]
\end{aligned}
$$

$X A-$ trials:

$$
\begin{aligned}
& \Delta V_{X}=\alpha B\left[0-\left(V_{X}+V_{A}\right)\right] \\
& \Delta V_{A}=\alpha B\left[0-\left(V_{X}+V_{A}\right)\right]
\end{aligned}
$$

$X+$ trials:

$$
\Delta V_{X}=\alpha B\left[1-V_{X}\right]
$$

$X-$ trials:

$$
\Delta V_{X}=\alpha B\left[0-V_{X}\right]
$$

The value of the actual outcome is 1 on trials with the outcome present and 0 on trials with the outcome absent. The value of the expected outcome is the sum of the predictive strengths of all cues present on that trial.

$V_{A}$ is altered on two different trial types. $V_{X}$ is altered on all four trial types. The values contained in the cells of the $2 \times 2$ table correspond to the frequencies of the four trial types. Thus, for any particular block of trials, the average change in $V_{A}$ is the sum of Equations 3 and 5, weighted for the relative frequencies of the two trial types. The average change in $V_{X}$ is the sum of Equations 2, 4, 6, and 7, weighted for the relative frequencies of the four trial types.

$$
\text { Mean } \Delta V_{A}=a \alpha B\left[1-\left(V_{X}+V_{A}\right)\right]+b \alpha B\left[0-\left(V_{X}+V_{A}\right)\right] \text {. }
$$

$$
\text { Mean } \begin{aligned}
\Delta V_{X}= & a \alpha B\left[1-\left(V_{X}+V_{A}\right)\right]+b \alpha B\left[0-\left(V_{X}+V_{A}\right)\right] \\
& +c \alpha B\left[1-V_{X}\right]+d \alpha B\left[0-V_{X}\right] .
\end{aligned}
$$

Equations 8 and 9 can be simplified to Equations 10 and 11 , respectively.

$$
\begin{gathered}
\text { Mean } \Delta V_{A} / \alpha B=a-V_{A}(a+b)-V_{X}(a+b) \\
\text { Mean } \Delta V_{X} / \alpha B=a+c-V_{A}(a+b)-V_{X}(a+b+c+d)
\end{gathered}
$$

Learning will progress until each of Equations 10 and 11 equals zero. At this point, $V_{A}$ and $V_{X}$ may be treated as constants. Although they may fluctuate from trial to trial, their means will maintain a constant value over many blocks of trials. $V_{A}$ is the predictive strength of the cue of interest. If the Rescorla-Wagner model is equivalent to the $\Delta p$ rule, then $V_{A}$ will equal $\Delta p$. that

Setting Equation 11 equal to zero and solving for $V_{X}$, we find

$$
V_{X}=\left[a+c-V_{A}(a+b)\right] /(a+b+c+d) .
$$

Setting Equation 10 equal to zero and substituting for $V_{X}$ using Equation 12, we find

$0=a-V_{A}(a+b)-(a+b)\left[a+c-V_{A}(a+b)\right] /(a+b+c+d)$.

Solving Equation 13 for $V_{A}$, we find that

$$
V_{A}=a /(a+b)-c /(c+d) .
$$

Equation 14 is the $\Delta p$ rule. Thus, in a contingency-judgment design involving only one cue, the predictive strength of that cue as described by the Rescorla-Wagner model is identical to the contingency as described by the $\Delta p$ rule.

(Manuscript received December 1, 1989; revision accepted for publication March 23, 1990.) 